\title{
Moderate wine consumption is associated with lower hemostatic and inflammatory risk factors over 8 years: The study of women's health across the nation (SWAN)
}

\author{
Imke Janssen*, Alan L. Landay, Kristine Ruppert and Lynda H. Powell \\ Department of Preventive Medicine, Rush University Medical Center, Chicago, IL, USA
}

\begin{abstract}
Moderate wine consumption has been associated with reduced cardiovascular (CV) risk, but most investigations have been conducted in Caucasian populations. To investigate the relationship of wine consumption to CV risk markers, we studied a multi-ethnic sample of middle-aged, healthy women $(N=2900 ; 48 \%$ white, $28 \%$ black, $7 \%$ Hispanic, $8 \%$ Chinese, $9 \%$ Japanese) participating in SWAN over 7 years with repeated assessments of CV risk factors. Consumption of wine was stable and common with $20 \%$ reporting none, $69 \%$ light ( $<1 /$ day), $7 \%$ moderate ( $=1 /$ day), and $4 \%$ heavy $(>1 /$ day). To guard against underreporting, we took the maximum reported wine consumption over 7 years as the predictor. We used mixed models with a random intercept and random time to assess the relationship between wine consumption and $\mathrm{CV}$ risk factors with moderate consumption as the reference. Outcome variables were log-transformed where necessary. Longitudinal models were adjusted for ethnicity, age, and time-varying menopausal status, hormone therapy use, overall alcohol consumption, high density lipoprotein (HDL), statin use, and a healthy lifestyle score based on physical activity, not smoking, and weight maintenance. Interactions of wine consumption with time were not significant. Moderate wine consumers had significantly lower levels of C-reactive protein $(\mathrm{CRP}, p<.001)$, fibrinogen $(p<.001)$, factor VII $(p<.01)$, and plasminogen activator inhibitor (PAI- $1, p<.05)$ than women who drank no or little wine. These associations were independent of significant effects of healthy lifestyle and overall alcohol consumption and similar across ethnic groups. Moderate wine consumption may protect against CVD via inflammatory and clotting pathways.
\end{abstract}

Keywords: Moderate wine consumption, inflammation, coagulation, longitudinal study, linear mixed models, midlife women

\section{Introduction}

In recent years, several meta-analyses [1-3] have been published on the effects of alcohol consumption and cardiovascular disease (CVD). Moderate consumption of any type of alcohol reduced overall mortality by $17 \%$ in men and by $18 \%$ in women [1]. The risk of incident coronary heart disease (CHD), CHD mortality, as well as CVD mortality was reduced by about $30 \%$ for women [2]. Dose-response analyses indi-

*Corresponding author: Imke Janssen, Department of Preventive Medicine, Rush University Medical Center, 1700W Van Buren, Suite 470, Chicago, IL 60612, USA. E-mail: Imke_Janssen@ rush.edu. cated that, compared to abstainers, the best protection is observed for moderate drinkers ( = 1glass/day for women) $[1,2]$, and that heavy drinkers are at increased risk. The J-shaped relation of alcohol consumption with fatal and non-fatal CV events was confirmed if the type of alcohol consumed was wine or beer but not liquor [3]. Furthermore, the maximal protection of $31 \%$ was observed for moderate wine drinkers.

The reduction in CVD risk for light to moderate alcohol drinkers is generally attributed to a beneficial effect on high density lipoprotein cholesterol (HDL) levels. However, the HDL hypothesis was disputed recently in a large, population based cohort study, with extensive control for confounding factors [4]. 
Inflammation is a critical component in many human diseases, including CVD, neurodegenerative diseases, diabetes, and some cancers. Multiple epidemiological studies have demonstrated the beneficial effects of light to moderate alcohol consumption on inflammatory markers with significantly decreased levels of plasma C-reactive protein (CRP) [5-7], factor VII [8], and fibrinogen $[5,8,9]$. Compared to women who drank no alcohol, women who consumed 1-2 drinks/day had 32\% lower CRP levels [10]. Whereas heavy alcohol consumption has been related to higher levels of plasminogen activator inhibitor antigen- 1 (PAI-1) and tissue plasminogen activator antigen (tPA) $[8,11]$, wine drinkers tend to have lower levels of these markers [8, 12].

These findings are consistent with the hypothesis that a balance between hemostatic and fibrinolytic activity may contribute to the complex relation of alcohol consumption with CHD [8, 13]. However, most previous studies of the relationship of alcohol consumption with hemostatic and inflammatory factors have been crosssectional $[5,8,10,13]$ or were restricted to Caucasians $[5,6,8,10,11,13]$. African American (but no other minority) women have been included in a few studies $[7,9]$. Furthermore, these studies consider only total alcohol consumption and not the type of beverage. In a meta-analysis of the effect of alcohol consumption in intervention studies [14], fibrinogen was significantly negatively associated with alcohol use whereas CRP, PAI-1, and tPA were not significantly related to alcohol use. Clinical trials have shown that CRP and fibrinogen are reduced after wine consumption [15]. Although there is benefit to the consumption of any alcohol in moderation, the effect of wine on cardiovascular health seems to be stronger. We hypothesized that moderate wine consumption is associated with (1) lower levels of hemostatic and inflammatory factors and (2) slower progression over time. We further hypoethized that (3) the associations are independent of ethnicity, socioeconomic status (SES), lifestyle factors, and total alcohol consumption.

\section{Methods}

\subsection{Study design and participants}

SWAN is a multi-ethnic, community-based, longitudinal cohort study of the natural history of the menopausal transition in 3302 women enrolled at seven sites throughout the United States: Boston, MA; Chicago, IL; Detroit, MI; Los Angeles, CA; Oakland, CA; Newark, NJ; and Pittsburgh, PA. The design of the main study has been reported [16]. The cohort included women of Caucasian, African-American, Chinese, Japanese, and Hispanic origins, aged 42-52 years who (1) had an intact uterus and at least 1 ovary, (2) had at least 1 menstrual period in the previous 3 months, (3) used no hormone therapy (HT) in the previous 3 months, and (4) were not pregnant. Women with a history of heart attack, stroke, or angina at study entry were excluded from the current analyses $(N=57)$. Women with no information on wine consumption $(N=241)$ and women with no assessment of hemostatic or inflammatory markers $(N=104)$ were excluded, leaving 2900 for the current analyses. Compared to eligible women excluded from the analyses, those who were included were less likely to be Hispanic, had higher SES and a healthier lifestyle (more physically active and less likely to smoke), were less likely to abstain from wine consumption, and had lower BMI. They did not differ in age, menopausal status, overall alcohol consumption, HDL, statin use, or any of the hemostatic or inflammatory factors. All analyses used data from baseline through the 7 th follow-up visit.

\subsection{Assessments}

All participants underwent annual exams which included interviews, anthropometry, questionnaires, and a blood draw for the assessment of all factors of interest including sociodemographic factors, reproductive hormones, and CV risk factors.

Outcome variables were CRP, factor VII activity, fibrinogen, PAI-1, and TPA measured in plasma. CRP was quantitated using an ultrasensitive rate immunonephelemetric method (Dade-Behring, Marburg, Germany). Fibrinogen and factor VII activity were measured in frozen citrated plasma (MLA ELECTRA 1400 C, Medical Laboratory Automation Inc., Mt. Vernon, NY) using a turbidometric detection system. PAI-1 was measured with a sandwich procedure using a solid phased monoclonal antibody and an enzyme labeled goat second antiserum for detection (IMUBIND plasma PAI-1 ELISA, American Diagnostica, Greenwich, CT). The tPA was measured in plasma using a double antibody in an enzyme linked immunosorbant assay (IMUBIND tPA ELISA, 
Table 1

Cardiovascular health

\begin{tabular}{llll}
\hline & Poor & Intermediate & Ideal \\
\hline Smoking & Current & Former $\leq 12$ mo & Never or former $>12$ mo \\
BMI $\left(\mathrm{kg} / \mathrm{m}^{2}\right)$ & $\geq 30$ & $25-29.9$ & $<25$ \\
Physical Activity & None & at least some moderate intensity & $\geq 120$ min/week moderate \\
Diet & 0 components* & 1 components* & $2-3$ components* $^{*}$ \\
\hline
\end{tabular}

$* \geq 4.5$ cups fruits and vegetables/day; $<1500 \mathrm{mg}$ sodium/day; $\geq 1.1 \mathrm{~g}$ of fiber per $10 \mathrm{~g}$ of carbohydrate.

American Diagnostica, Greenwich, CT). The assay uses human single chain tPA as a standard calibrated against an international standard (NIBSAC, Hertfordshire, UK). Because of budgetary constraints, CRP, PAI-1, and tPA were assayed annually with the exception of follow-up visit 2; fibrinogen and factor VII activity were assayed at baseline and odd follow-up years only. Consistent with published guidelines, CRP values $>10$ were excluded (8.6\% of observations). For all other markers, to identify extreme values (probably reflecting acute illness), values outside \pm 3 SD were excluded ( $<2 \%$ for each marker). Because of different assessment schedules and exclusion criteria, sample size for the analyses varied from 2811 to 2886 women; the total number of observations used in the longitudinal analyses ranged from 9905 to 15289 .

Diet, including consumption of fruits and vegetables, sodium, fiber, carbohydrates, and total alcohol, was assessed with a food frequency questionnaire (FFQ) [17] at baseline and visit 5. Wine, beer, and liquor consumption was assessed in all years when the complete FFQ was not assessed. For each type of alcohol, there were 9 possible responses: none or less than one per month, 1-3 per month, 1 per week, 2-4 per week, 5-6 per week, 1 per day, 2-3 per day, 4 per day, 5 or more per day. Wine consumption was condensed into categories of none, $<1$ glass/day, = 1 glass/day, or $>1$ glass/day. Since wine consumption was stable over time for the women with repeat assessments [18], and to guard against underreporting, we took the maximum reported wine consumption over the study period as the main predictor.

Total alcohol consumption was classified as none ( $<1$ drink/month), moderate ( $>1 /$ month, $\leq 1 /$ week), and high $(>1 /$ week), providing a time-varying covariate. Physical activity was assessed with an adapted version of the Kaiser Physical Activity Survey (KPAS) [19] and classified as in previous SWAN publications [20, 21], see Table 1.

Smoking status was assessed every year by selfreport. Standardized protocols were used to measure height and weight. Height was measured without shoes using a stadiometer. Weight was measured without shoes and with light indoor clothing using scales calibrated to a standard on a monthly basis. Body mass index (BMI) was calculated as weight in kilograms divided by height in meters squared. A healthy lifestyle score based on physical activity, not smoking, weight maintenance, and diet was created using guidelines developed by the American Heart Association [22] for cardiovascular health. Each attribute was classified as ideal, intermediate or poor (Table 1). We created a healthy lifestyle score by assigning 1 point for each health behavior in the intermediate category and 2 points in the ideal category. Higher scores (range 0-8) indicated a healthier lifestyle. The score was calculated at each visit using a simple update method for physical activity and diet as used in the Nurses' Health Study [23].

HDL was analyzed on EDTA-treated plasma and isolated using heparin-2 $\mathrm{M}$ manganese chloride [24]. Education ( $\leq$ high school degree, some college/vocational training, college degree, or more) was assessed at baseline. Current smoking status, family income, menopausal status, and medication use including HT was assessed by questionnaire annually.

\subsection{Statistical methods}

Baseline characteristics of the sample were summarized as mean (SD) or $\mathrm{N}(\%)$ and compared to women excluded from the study using the two-sample $t$-test or the chi-square test, respectively. Because of their skewed distribution CRP, PAI-1, and tPA were transformed by natural logarithm, and summarized as geometric mean (95\% confidence interval).

We used linear mixed models with time from baseline to time of assessment modeled continuously and wine consumption as the primary predictor (with moderate consumption as the reference). A random intercept was included to account for different 
baseline values; time was also included as a random effect to allow for different trajectories for each woman. Assumptions were examined using residuals. A heterogeneous autoregressive covariance structure provided the best fit in all analyses according to the Bayes Information Criterion (BIC). Model 1 adjusted for age at baseline, site, and ethnicity. Model 2 further adjusted for SES (education $\leq$ high school) and time-varying covariates of total alcohol consumption, healthy lifestyle, HDL cholesterol, statin use, menopausal status, and HT use. For logtransformed outcomes, regression coefficients were back-transformed using the formula $[100(\exp (\beta)-1)]$ to calculate the percent change ( $\%$ change) in the outcome per unit change in the predictor [25]. To test whether time trends differed by wine consumption, an interaction term of time and wine consumption was included. These interactions were not significant and are therefore not reported here. Sensitivity analyses included (1) using a time-varying indicator of SES (income), (2) using BMI and smoking (instead of the healthy lifestyle score), (3) excluding Hispanic women, and (4) using wine consumption as a timevarying predictor.

\section{Results}

Table 2 shows the baseline characteristics of the analytic cohort in 1996/97. Almost half of the women were Caucasian, $28 \%$ were black, and the remaining women were about equally from the other 3 ethnicities (Hispanic, Chinese, and Japanese). The average age was 46 years; $54 \%$ were pre-menopausal, $46 \%$ were early peri-menopausal, and $16 \%$ were smokers. Average BMI was $28.1 \mathrm{~kg} / \mathrm{m}^{2}$; more than $60 \%$ had a poor diet, and only $4 \%$ followed a healthy diet, $44 \%$ did not engage in physical activity, and almost half reported low total alcohol consumption. Consumption of wine was stable and common with $20 \%$ reporting none, $69 \%$ light $(<1 /$ day), $7 \%$ moderate ( $=1 /$ day $)$, and $4 \%$ heavy (>1/day). At baseline, hemostatic and inflammatory markers were in the normal range.

Table 3 shows numerical results, and Fig. 1 provides a graphical illustration of the longitudinal analyses. Highly significantly changes over time were observed in all markers (all $p<.001$ ). In minimally adjusted models, CRP increased by $2.4 \%$ year; PA-1 decreased by $7.0 \% / y e a r$, and tPA decreased by $1.0 \%$ year. Factor VII increased by 1.6 percentage points annually, and fibrinogen decreased by $1.9 \mathrm{mg} / \mathrm{l}$ per year. In fully adjusted models, the estimated time trends were slightly attenuated but remained highly significant. Moderate wine consumers had significantly lower levels of CRP and factor VII compared to women who drank no or little wine $(<1 /$ day) in minimally adjusted models. These differences were attenuated but remained significant in fully adjusted models $(p<.05)$. Fibrinogen showed a monotone relationship to wine consumption with the lowest levels observed in women who drank $>1 /$ day. In the minimally adjusted model, all pairwise group comparisons were significant $(p<.01)$. In the fully adjusted model, these differences were attenuated, and moderate wine drinkers had lower levels than abstainers and higher levels than women who drank $>1 /$ day (both $p<.05$ ). PAI-1 and tPA levels were significantly lower in moderate wine consumers compared to all other groups in minimally adjusted analyses, but after full adjustment, only women who drank $>1$ /day had significantly higher levels than moderate consumers $(p<.001)$.

In all analyses, age at baseline was positively and healthy lifestyle was negatively associated with all hemostatic and inflammatory factors. Associations with other covariates were less consistent. HDL was negatively associated with CRP, fibrinogen, PAI-1, and tPA, but positively related to factor VII. Postmenopausal women had higher levels of factor VII and fibrinogen and lower levels of tPA, and HT use was related to higher CRP and factor VII and lower PAI-1. Statin use was related to lower CRP and PAI-1 levels and to higher fibrinogen. Total alcohol consumption was unrelated to CRP and factor VII, but inversely related to fibrinogen, PAI-1, and tPA.

\section{Discussion}

In this large longitudinal study of women, moderate wine consumers ( $=1$ glass/day) had significantly lower levels of all hemostatic and inflammatory factors examined compared to abstainers. The effect of wine consumption on CRP and factor VII exhibited an inverse J-shape; moderate wine drinkers had significantly lower levels of these markers than women who drank no or little wine $(<1 /$ day). The relation of wine to fibrinogen was monotone with abstainers having the highest level and women drinking $>1$ /day having the lowest level. In contrast, PAI-1 and tPA were highest for women drinking $>1$ glass of wine per day with no 
Table 2

Characteristics of the Cohort at Baseline

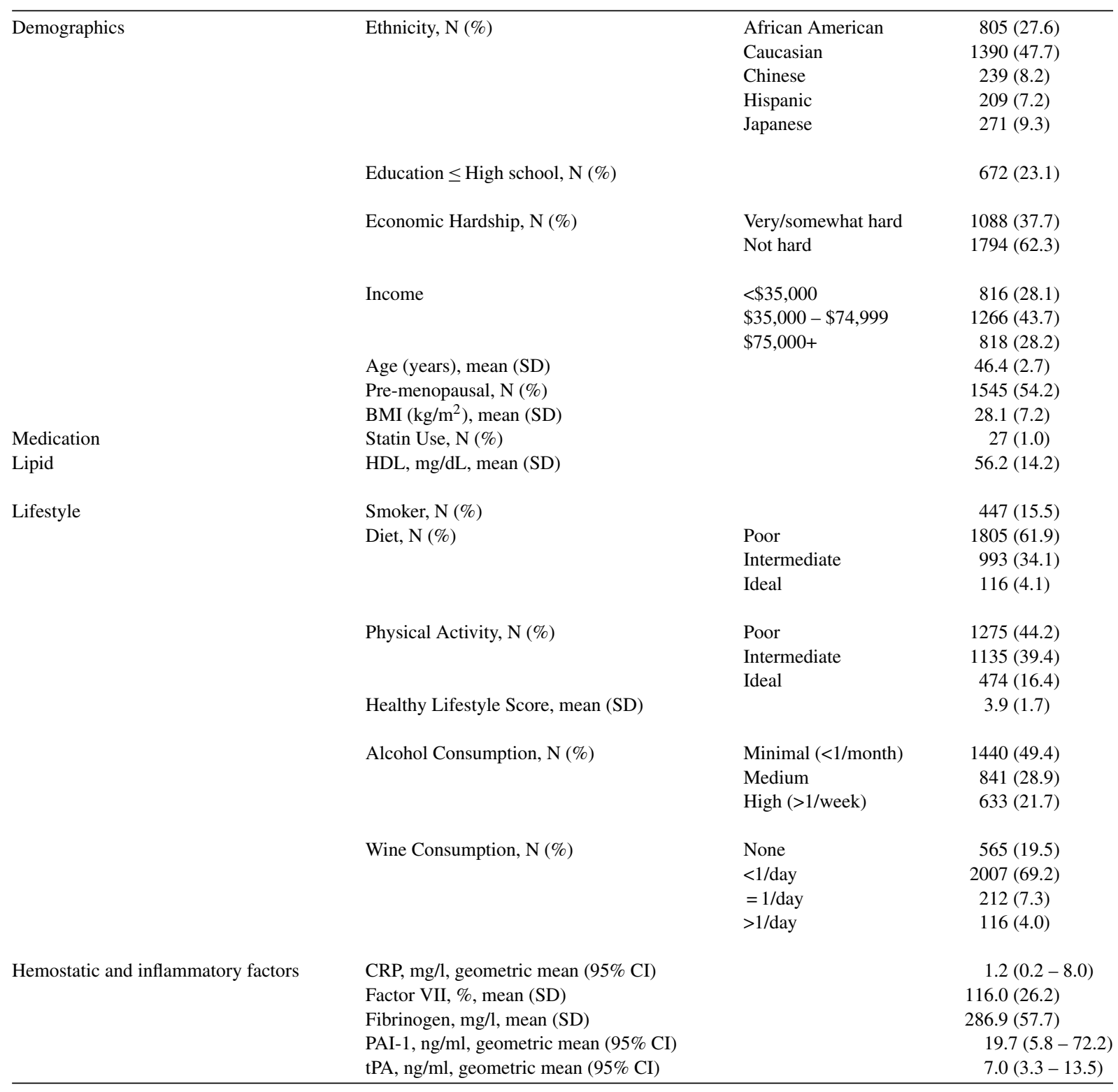

significant differences between the other 3 groups in the fully adjusted analyses. These results are similar to those for women from the Framingham Offspring Study, where the relationship of wine consumption with fibrinogen was linear, whereas factor VII, PAI1 and tPA were better fit by the addition of a quadratic term [8]; the association with CRP was not reported in that cross-sectional study of Caucasians.

This cohort of middle-aged women was healthy but heavy with BMI in the overweight range. At baseline, hemostatic and inflammatory markers were in the normal range and changed significantly over time. These trends were similar to those estimated in an earlier analysis of SWAN [26] which used fewer follow-up visits. Excluding Hispanic women resulted in slightly stronger effects of moderate wine consumption in sensitivity analyses. Using a time-varying indicator of SES (i.e. income) did not alter results. Using BMI and smoking instead of the healthy lifestyle score yielded similar and only slightly weaker effects of wine 
Table 3

The relationship of time and wine consumption to hemostatic and inflammatory markers

\begin{tabular}{|c|c|c|c|c|c|}
\hline outcome & & Minimally adjusted $^{\wedge}$ & & Fully adjusted $\ddagger$ & \\
\hline \multirow[t]{5}{*}{$\mathrm{CRP}(\mathrm{mg} / \mathrm{l})$} & Time (year) & $2.4(1.9,3.0)$ & $* * *$ & $2.0(1.4,2.6)$ & $* * *$ \\
\hline & $>1 /$ day & $3.8(-16.7,29.4)$ & & $3.5(-15.0,26.1)$ & \\
\hline & None & $51.9(29.3,78.4)$ & $* * *$ & $24.8(7.3,45.0)$ & $* *$ \\
\hline & $<1 /$ day & $40.4(22.1,61.3)$ & $* * *$ & $18.2(4.0,34.4)$ & $*$ \\
\hline & = 1/day $($ Reference $)$ & - & & - & \\
\hline \multirow[t]{5}{*}{ Factor VII (\%) } & Time (year) & $1.6(1.4,1.7)$ & $* * *$ & $1.2(1.0,1.4)$ & $* * *$ \\
\hline & $>1 /$ day & $4.2(-0.8,9.2)$ & & $3.2(-1.8,8.1)$ & \\
\hline & None & $7.1(3.4,10.7)$ & $* * *$ & $6.4(2.6,10.2)$ & $* * *$ \\
\hline & $<1 /$ day & $5.2(2.1,8.4)$ & $* * *$ & $4.9(1.7,8.2)$ & $* *$ \\
\hline & = 1/day $($ Reference $)$ & - & & - & \\
\hline \multirow[t]{5}{*}{ Fibrinogen (mg/l) } & Time (year) & $-1.9(-2.2,-1.6)$ & $* * *$ & $-1.9(-2.3,-1.5)$ & $* * *$ \\
\hline & $>1 /$ day & $-14.3(-24.5,-4.1)$ & $* *$ & $-12.4(-22.0,-2.7)$ & $*$ \\
\hline & None & $21.0(13.6,28.4)$ & $* * *$ & $8.5(1.0,16.1)$ & $*$ \\
\hline & $<1 /$ day & $14.8(8.3,21.2)$ & $* * *$ & $5.0(-1.4,11.4)$ & \\
\hline & $=1 /$ day $($ Reference $)$ & - & & - & \\
\hline \multirow[t]{5}{*}{ PAI-1 (ng/ml) } & Time (year) & $-7.0(-7.4,-6.5)$ & $* * *$ & $-6.6(-7.1,-6.1)$ & $* * *$ \\
\hline & $>1 /$ day & $19.4(2.5,39.0)$ & $*$ & $23.7(8.3,41.3)$ & $* *$ \\
\hline & None & $25.8(12.6,40.5)$ & $* * *$ & $10.4(-0.4,22.4)$ & \\
\hline & $<1 /$ day & $19.2(8.2,31.2)$ & $* * *$ & $7.1(-1.9,16.9)$ & \\
\hline & = 1/day (Reference) & - & & - & \\
\hline \multirow[t]{5}{*}{ tPA (ng/ml) } & Time (year) & $-1.0(-1.2,-0.7)$ & $* * *$ & $-0.6(-0.9,-0.3)$ & $* * *$ \\
\hline & $>1 /$ day & $12.8(4.4,21.9)$ & $* *$ & $14.0(6.6,21.8)$ & $* * *$ \\
\hline & None & $7.2(1.3,13.4)$ & $*$ & $0.5(-4.6,5.9)$ & \\
\hline & $<1 /$ day & $5.1(0.1,10.4)$ & $*$ & $-0.8(-5.1,3.7)$ & \\
\hline & $=1 /$ day $($ Reference $)$ & - & & - & \\
\hline
\end{tabular}

${ }^{*} p<.05,{ }^{* *} p<.01,{ }^{* * *} p<.001$ compared to $=1 /$ day; ${ }^{\wedge}$ adjusted for age at baseline, site, ethnicity; $\ddagger$ further adjusted for socioeconomic status, menopausal status, HT use, HDL cholesterol, statin use, healthy lifestyle, and total alcohol consumption.

consumption. Using time-varying wine consumption, on the other hand, weakened the observed associations, possibly because of under-reporting.

Mechanisms responsible for the healthful effects of wine are complex. Moderate intake of any alcoholic beverage produces positive effects on antioxidants, lipids, and platelets [27]. Wine demonstrates beneficial properties that are independent of alcohol consumption, which has been attributed to the polyphenol content of wine [28].

The alcohol component of wine may be responsible for its antiplatelet activities [29, 30]. Alcohol has anticoagulant properties that limit blood clotting by making platelets less prone to stick together and reducing the levels of fibrin protein that binds them together. Clinical trials have shown that in addition to wine, grapes, and grape juice can decrease platelet aggregation [31, 32]. The benefits of alcohol and polyphenols may be additive, since alcohol, red wine, and polyphenolic grape extract each inhibit platelet adhesion in human blood [33].

There is evidence that the effects of wine on blood coagulation may only be transient, indicating that moderate consistent consumption is critical for beneficial outcomes $[34,35]$. This could explain conflicting data from observational and intervention studies regarding the effect of wine on levels of CRP, factor VII, fibrinogen, tPA, and PAI-1 [14]. Inconsistencies in diet (i.e. high fat vs. low fat) between studies may also confound the results [36].

Strengths of the current study include the simultaneous assessment of five different biomarkers, the longitudinal design, the large, ethnically diverse cohort with repeated assessments over 8 years of both predictor and outcome variables, and the well characterized covariates known to be related to hemostatic 

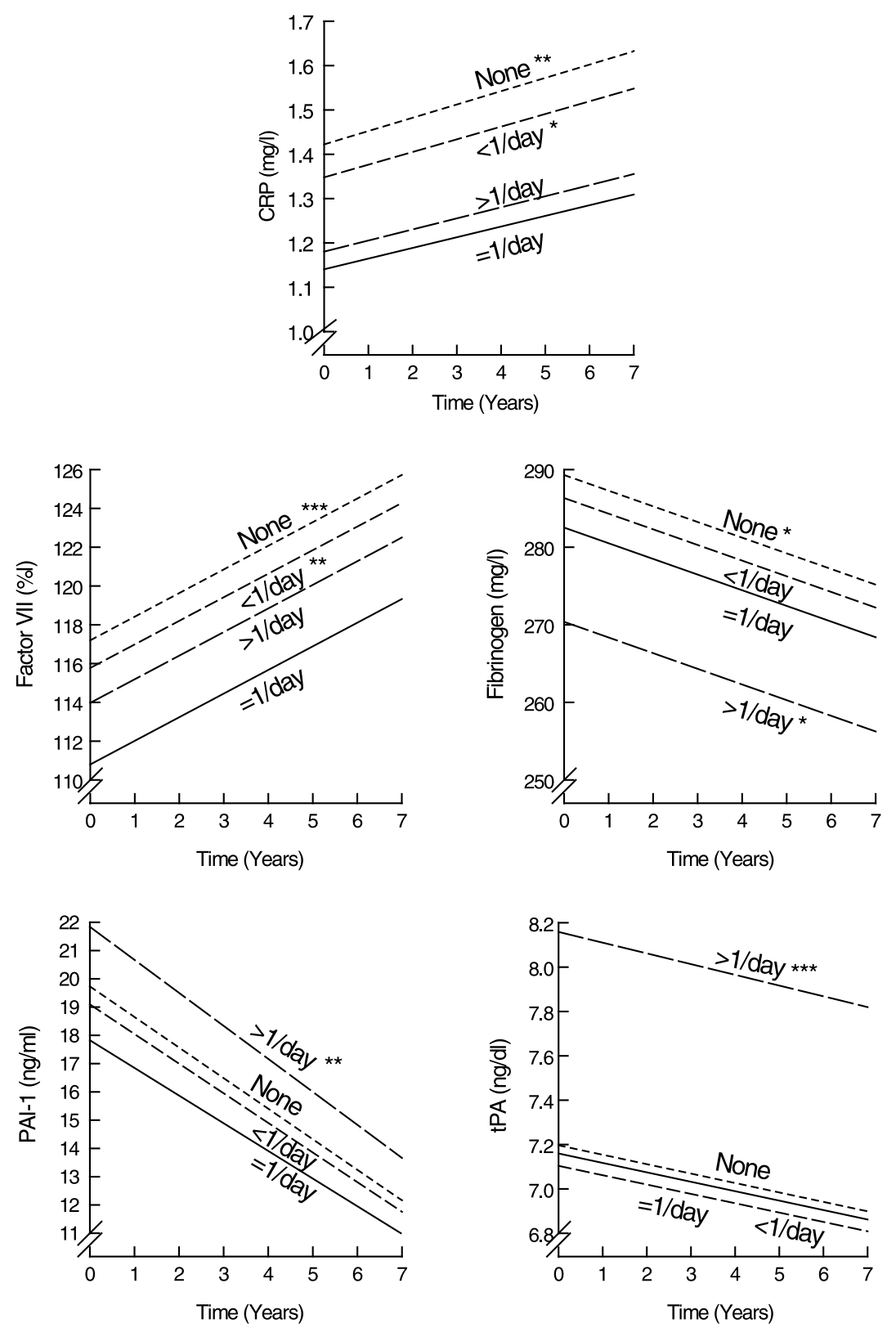

Fig. 1. Hemostatic and inflammatory markers over 8 years by wine consumption; ${ }^{*} p<.05,{ }^{* *} p<.01,{ }^{* * *} p<.001$ compared to $=1 /$ day; ${ }^{\wedge}$ adjusted for age at baseline, site, ethnicity; ${ }^{\ddagger}$ further adjusted for socioeconomic status, menopausal status, HT use, HDL cholesterol, statin use, healthy lifestyle, and total alcohol consumption.

and inflammatory markers. Limitations of the study include that only $69 \%$ of women were retained in the analysis by the last assessment. The analytic approach, however, is robust against missing data in estimating the effect of wine consumption on markers. Another limitation is the fairly low number of women who consumed a moderate amount of wine, especially among
non-Caucasians. Therefore, subgroup analyses were not feasible. However, the total number of women in each ethnic group was several hundred, and observed relationships were consistent.

In summary, we found that women who consumed wine in moderation had the lowest levels of all hemostatic and inflammatory factors, independent of age, 
ethnicity, socioeconomic status, as well as significant covariates such as HT use, HDL, healthy lifestyle, and total alcohol consumption. In conclusion, moderate wine consumption may protect against CVD via inflammatory and clotting pathways.

\section{Acknowledgments}

The Study of Women's Health Across the Nation (SWAN) has grant support from the National Institutes of Health (NIH), DHHS, through the National Institute on Aging (NIA), the National Institute of Nursing Research (NINR) and the NIH Office of Research on Women's Health (ORWH) (Grants U01NR004061; U01AG012505, U01AG012535, U01AG012531, U01AG012539, U01AG012546, U01AG012553, U01AG012554, U01AG012495). The content of this paper is solely the responsibility of the authors and does not necessarily represent the official views of the NIA, NINR, ORWH or the NIH.

Clinical Centers: University of Michigan, Ann ArborSiobán Harlow, PI 2011 - present, MaryFran Sowers, PI 1994-2011; Massachusetts General Hospital, Boston, MA - Joel Finkelstein, PI 1999 - present; Robert Neer, PI 1994 - 1999; Rush University, Rush University Medical Center, Chicago, IL - Howard Kravitz, PI 2009 - present; Lynda Powell, PI 1994 2009; University of California, Davis/Kaiser - Ellen Gold, PI; University of California, Los Angeles - Gail Greendale, PI; Albert Einstein College of Medicine, Bronx, NY - Carol Derby, PI 2011 - present, Rachel Wildman, PI 2010 - 2011; Nanette Santoro, PI 20042010; University of Medicine and Dentistry - New Jersey Medical School, Newark - Gerson Weiss, PI 19942004; and the University of Pittsburgh, Pittsburgh, PA - Karen Matthews, PI.

NIH Program Office: National Institute on Aging, Bethesda, MD - Winifred Rossi 2012 -present; Sherry Sherman 1994 - 2012; Marcia Ory 1994 - 2001; National Institute of Nursing Research, Bethesda, $M D$ - Program Officers.

Central Laboratory: University of Michigan, Ann Arbor - Daniel McConnell (Central Ligand Assay Satellite Services).

Coordinating Center: University of Pittsburgh, Pittsburgh, PA-Maria Mori Brooks, PI 2012 - present; Kim
Sutton-Tyrrell, PI 2001 -2012; New England Research Institutes, Watertown, MA - Sonja McKinlay, PI 1995 2001.

Steering Committee: Susan Johnson, Current Chair; Chris Gallagher, Former Chair

We thank the study staff at each site and all the women who participated in SWAN.

\section{References}

[1] Di Castelnuovo A, Costanzo S, Bagnardi V, Donati MB, Iacoviello L, de Gaetano G. Alcohol dosing and total mortality in men and women: An updated meta-analysis of 34 prospective studies. Arch Intern Med 2006;166(22):2437-2445.

[2] Ronksley PE, Brien SE, Turner BJ, Mukamal KJ, Ghali WA. Association of alcohol consumption with selected cardiovascular disease outcomes: A systematic review and meta-analysis. BMJ 2011;342:d671.

[3] Costanzo S, Di Castelnuovo A, Donati MB, Iacoviello L and de Gaetano G. Wine, beer or spirit drinking in relation to fatal and non-fatal cardiovascular events: A meta-analysis. Eur J Epidemiol 2011;26(11):833-50.

[4] Magnus P, Bakke E, Hoff DA, Hoiseth G, Graff-Iversen S Knudsen GP. et al. Controlling for high-density lipoprotein cholesterol does not affect the magnitude of the relationship between alcohol and coronary heart disease. Circulation 2011;124(21):2296-302.

[5] Imhof A, Woodward M, Doering A, Helbecque N, Loewel $\mathrm{H}$, Amouyel P. et al. Overall alcohol intake, beer, wine, and systemic markers of inflammation in western Europe: Results from three MONICA samples (Augsburg, Glasgow, Lille). Eur Heart J 2004;25(23):2092-100.

[6] Raum E, Gebhardt K, Buchner M, Schiltenwolf M, Brenner H. Long-term and short-term alcohol consumption and levels of C-reactive protein. Int J Cardiol 2007;121(2): 224-6.

[7] Volpato S, Pahor M, Ferrucci L, Simonsick EM, Guralnik JM, Kritchevsky SB. et al. Relationship of alcohol intake with inflammatory markers and plasminogen activator inhibitor-1 in well-functioning older adults: The Health, Aging, and Body Composition study. Circulation 2004;109(5):607-12.

[8] Mukamal KJ, Jadhav PP, D’Agostino RB, Massaro JM, Mittleman MA, Lipinska I. et al. Alcohol consumption and hemostatic factors: Analysis of the Framingham Offspring cohort. Circulation 2001;104(12):1367-73.

[9] Mukamal KJ, Cushman M, Mittleman MA, Tracy RP, Siscovick DS. Alcohol consumption and inflammatory markers in older adults: The Cardiovascular Health Study. Atherosclerosis 2004;173(1):79-87.

[10] Pai JK, Hankinson SE, Thadhani R, Rifai N, Pischon T, Rimm EB. Moderate alcohol consumption and lower levels of inflammatory markers in US men and women. Atherosclerosis, 2006 5;186(1):113-120.

[11] Djousse L, Pankow JS, Arnett DK, Zhang Y, Hong Y, Province MA. et al. Alcohol consumption and plasminogen activator inhibitor type 1: The national heart, lung, and blood institute family heart study. Am Heart J 2000; 139(4):704-9. 
[12] Rimm EB, Williams P, Fosher K, Criqui M and Stampfer MJ. Moderate alcohol intake and lower risk of coronary heart disease: Meta-analysis of effects on lipids and hemostatic factors. BMJ 1999;319(7224): 1523-8.

[13] Mukamal KJ, Massaro JM, Ault KA, Mittleman MA, Sutherland PA, Lipinska I. et al. Alcohol consumption and platelet activation and aggregation among women and men: The framingham offspring study. Alcohol Clin Exp Res 2005;29(10):1906-12.

[14] Brien SE, Ronksley PE, Turner BJ, Mukamal KJ, Ghali WA. Effect of alcohol consumption on biological markers associated with risk of coronary heart disease: Systematic review and meta-analysis of interventional studies. BMJ 2011;342:d636.

[15] Leighton F, Urquiaga I. Changes in Cardiovascular Risk Factors Associated with wine consumption in intervention studies in humans. ann Epidemiol 2007;17(5 Supplement):S32-S36.

[16] Sowers MF, Crawford S, Sternfeld B, SWAN: A Multicenter Multiethnic, Community-Based Cohort Study of Women and the Menopausal Transition. In: LObo RA, Kelsey J, Marcus R, editors. Menopause: Biology and Pathobiology. San Diego, Calif.; London: Academic; 2000. p. 175-88.

[17] Block G, Hartman AM, Dresser CM, Carroll MD, Gannon J, Gardner L. A data-based approach to diet questionnaire design and testing. Am J Epidemiol 1986;124(3):453-69.

[18] Powell LH, Janssen I, Wesley D. Stability of moderate wine consumption over seven years in middle-aged women. Proceedings of the 3rd Interbational Congress on Wine and Health 2007.

[19] Sternfeld B, Ainsworth BE, Quesenberry CP. Physical activity patterns in a diverse population of women. Prev Med 1999;28(3):313-23.

[20] Dugan SA, Everson-Rose SA, Karavolos K, Avery EF, Wesley DE, Powell LH Physical Activity, Reduced. Intra-abdominal Fat in Midlife African-American and White Women. Obesity 2010;18:1260-5.

[21] Janssen I, Dugan SA, Karavolos K, Lynch EB, Powell LH. Correlates of 15-Year Maintenance of Physical Activity in Middle-Aged Women. Int J Beh Med 2013; epub ahead of print.

[22] Lloyd-Jones DM, Hong Y, Labarthe D, Mozaffarian D, Appel LJ, Van Horn L. et al. Defining and setting national goals for cardiovascular health promotion and disease reduction: The American Heart Association's strategic Impact Goal through 2020 and beyond. Circulation 2010;121(4):586-613.

[23] Tworoger SS, Gertig DM, Gates MA, Hecht JL, Hankinson SE. Caffeine, alcohol, smoking, and the risk of incident epithelial ovarian cancer. Cancer 2008;112(5):1169-77.
[24] Warnick GR and Albers JJ. A comprehensive evaluation of the heparin-manganese precipitation procedure for estimating high density lipoprotein cholesterol. J Lipid Res 1978;19(1):65-76.

[25] Singer JD, Willett JB. Applied longitudinal data analysis: Modeling change and event occurrence: Modeling change and event occurrence : Oxford University Press, USA; 2003.

[26] Matthews KA, Schott LL, Bromberger J, Cyranowski J, Everson-Rose SA, Sowers MF. Associations between depressive symptoms and inflammatory/hemostatic markers in women during the menopausal transition. Psychosom Med 2007;69(2):124-130.

[27] Lindberg ML, Amsterdam EA. Alcohol, wine, and cardiovascular health. Clin Cardiol 2008;31(8):347-51.

[28] Ruf J. Alcohol, wine and platelet function. Biol Res 2004;37(2):209-16.

[29] Pace-Asciak CR, Rounova O, Hahn SE, Diamandis EP, Goldberg DM. Wines and grape juices as modulators of platelet aggregation in healthy human subjects. Clinica chimica acta 1996;246(1):163-82.

[30] Pellegrini N, Pareti F, Stabile F, Brusamolino A, Simonetti P. Effects of moderate consumption of red wine on platelet aggregation and hemostatic variables in healthy volunteers. Eur J Clin Nutr 1996;50(4):209-13.

[31] Folts JD. Antithrombotic potential of grape juice and red wine for preventing heart attacks. Pharmaceutical Biology (Formerly International Journal of Pharmacognosy) 1998;36(Supplement 1):21-7.

[32] Keevil JG, Osman HE, Reed JD, Folts JD. Grape juice, but not orange juice or grapefruit juice, inhibits human platelet aggregation. J Nutr 2000;130(1):53-6.

[33] Lange DD, Scholman W, Kraaijenhagen R, Akkerman J and Van De Wiel A. Alcohol and polyphenolic grape extract inhibit platelet adhesion in flowing blood. Eur J Clin Invest 2004;34(12):818-24.

[34] Lavy A, Fuhrman B, Markel A, Dankner G, Ben-Amotz A, Presser D. et al. Effect of dietary supplementation of red or white wine on human blood chemistry, hematology and coagulation: Favorable effect of red wine on plasma high-density lipoprotein. Annals of Nutrition and Metabolism 1994;38(5):287-94.

[35] Kikura M, Levy JH, Safon RA, Lee MK, Szlam F. The influence of red wine or white wine intake on platelet function and viscoelastic property of blood in volunteers. Platelets 2004;15(1):37-41.

[36] Mezzano D. Distinctive effects of red wine and diet on hemostatic cardiovascular risk factors. Biol Res 2004; 37(2):217-24. 